\title{
Voiding function after sacrocolpopexy versus native tissue transvaginal repair for apical pelvic organ prolapse in an ERAS era: A retrospective cohort study
}

\author{
Amr Sherif El Haraki ${ }^{1}$ - Jersey Burns ${ }^{2} \cdot$ Christopher L. Crafton $^{1} \cdot$ Candace Parker-Autry ${ }^{1} \cdot$ Catherine Ann Matthews $^{1,2}$
}

Received: 23 June 2021 / Accepted: 4 September 2021 / Published online: 29 September 2021

(C) The International Urogynecological Association 2021

\begin{abstract}
Objective The objectives of this study were to compare time to return of voiding function and associated complications in women undergoing minimally invasive sacrocolpopexy (SCP) versus transvaginal native tissue repair in patients with same-day or early discharge.

Methods This was a retrospective cohort study conducted at a tertiary care center. The electronic medical record system was queried for women who underwent native tissue vaginal repair or SCP for apical prolapse between March and December 2020 using CPT codes for sacrocolpopexy (57425), extraperitoneal (57282), and intraperitoneal colpopexy (57283). Voiding success was our primary outcome and was defined by a postvoid residual $<150 \mathrm{ml}$. Secondary outcomes included catheter days and urinary tract infections. The total number of participants was based on a power calculation using internal institutional rates. Participants were compared based on the surgical approach. A multivariate regression analysis was performed to assess for confounding factors.

Results We included 134 women: $63 \mathrm{SCP}$ and 71 native tissue. The failure rate of the first postoperative voiding trial was 34 vs. 11\% (odds ratio: $4.91 ; 95 \%$ CI 1.96-12.3, $p<0.01$ ) in the vaginal and SCP groups, respectively. Both groups had a similar success rate of a second voiding trial (100\% in SCP group vs. $95.7 \%$ in the vaginal repair group, $p=1)$. The total number of days ( 3.108 vs. 1.603 days, $p<0.01)$ to return of bladder function, postoperative urinary tract infections $(23.9$ vs. $6.35 \%, p<0.01)$ and emergency department visits $(15.5$ vs. $1.59 \%, p<0.01)$ were all higher in the vaginal repair group.

Conclusions Vaginal apical native tissue repair had a fivefold greater risk of acute postoperative urinary retention compared to sacrocolpopexy in addition to increased rates of post-operative urinary tract infection and emergency department visits for urinary tract concerns.
\end{abstract}

Keywords Urinary retention $\cdot$ ERAS $\cdot$ Sacrocolpopexy $\cdot$ Voiding trial $\cdot$ Uterosacral $\cdot$ Sacrospinous $\cdot$ Native tissue repair $\cdot$ Voiding function

\section{Introduction}

Rates of postoperative incomplete bladder emptying after pelvic reconstructive surgery have a wide reported variance that ranges from $13 \%$ [1] to $39 \%$ [2]. Factors affecting

Catherine Ann Matthews

camatthe@wakehealth.edu

1 Department of Urology, Wake Forest Baptist Medical Center, 1 Medical Center Blvd, Winston-Salem, NC 27157, USA

2 Department of Obstetrics and Gynecology, Wake Forest Baptist Medical Center, 1 Medical Center Blvd, Winston-Salem, NC 27157, USA postoperative urinary retention (POUR) include the timing of the voiding trial [3], method of prolapse repair [4], concomitant hysterectomy, larger cystoceles, severe intra-operative blood loss, levator plication, and Kelly plication [5].

With enhanced recovery after surgery (ERAS) protocols widely enacted in female pelvic reconstructive surgery [6], same-day discharge has become a common phenomenon. While safe and cost effective for patients with no increase in postoperative complications as compared to an overnight stay [6-8], ERAS necessitates earlier attempts at voiding function and thus information about POUR for these patients is important. Discharge from the hospital with need for catheter drainage increases patient burden and may decrease overall 
satisfaction [9]. It is also associated with urinary tract infection and increased cost of care [10].

One potential mechanism by which POUR occurs following female pelvic reconstructive surgery is disruption of the local autonomic nervous system, increasing sympathetic drive to the bladder outlet and inhibiting urethral relaxation and bladder emptying [11]. Pain can also adversely impact voiding function [12]. Surgical approaches to prolapse repair, therefore, could impact the incidence of POUR [13]. Differences in POUR in women undergoing transvaginal native tissue repair versus minimally invasive sacrocolpopexy has only been investigated in a single trial [4] that reported a threefold higher risk in the native tissue group.

The objective of this study, therefore, was to test the hypothesis that minimally invasive SCP is associated with lower rates of POUR than transvaginal apical native tissue repair procedures when voiding trials are performed prior to hospital discharge within $24 \mathrm{~h}$ post-procedure. Our secondary objectives were to compare the number of days of catheter drainage and any associated complications within the first 30 days post procedure. This information may help facilitate postoperative planning as well as manage patient expectations and better aid in patient education regarding their anticipated postoperative course.

\section{Materials and methods}

This study was approved by the Wake Forest School of Medicine Institutional Review Board (IRB00071159). We performed a chart review and retrospective cohort study of women undergoing apical pelvic organ prolapse repair at Wake Forest Baptist Medical Center between March 2020 (month of ERAS initiation) and December 2020 (end of quality improvement period). This timeframe was chosen since our institution had globally adopted ERAS and same-day or early discharge starting in March 2020 as part of the response to the COVID-19 pandemic and decreasing exposure to the inpatient setting. Eligible women were older than 21 years at the time of surgery and underwent apical repair by laparoscopic/robotic sacrocolpopexy (CPT 57425), extraperitoneal vaginal colpopexy (CPT 57282), or intraperitoneal vaginal colpopexy (CPT 57283) for stage II or greater symptomatic pelvic organ prolapse. Patients were excluded if they underwent a concomitant colorectal procedure (i.e., rectopexy); had a history of preoperative urinary retention requiring catheterization; underwent an anterior or posterior repair without apical suspension; or had an intraoperative complication or surgical planning necessitating that their first postoperative voiding trial occur on a day greater than postoperative day 1 such as intraoperative urinary tract injury. Eligible women were divided into two groups for comparison: minimally invasive sacrocolpopexy versus apical native tissue vaginal repair. Our primary outcome was the pass rate of the first postoperative voiding trial. Secondary outcomes included days of catheter use, urinary tract infections, and emergency department visits for urinary tract related problems within the first 30 days of surgery. The total days of catheterization were assessed through postoperative provider and nursing notes that commented on return to normal voiding without need for intermittent or indwelling catheterization.

All women during the study period participated in a standardized ERAS protocol that included preoperative hydration, acetaminophen, ibuprofen, and Pyridium (phenazopyridine). Postoperatively, we continued the same pain regimen and added ice to the perineum; Tramadol and antiemetics were added as needed. More potent narcotics were avoided but were not prohibited. Our goal was same-day discharge to home or 23-h post-operative admission for observation.

All procedures were performed under the direct supervision of two attending female pelvic medicine trained surgeons. Trainees were present and active in every case. Minimally invasive sacrocolpopexy procedures were performed laparoscopically or robotically. Transvaginal apical repair procedures were performed using the uterosacral and sacrospinous ligaments according to attending preference for each individual case. Concomitant hysterectomy, mid-vaginal repair procedures (i.e., anterior and posterior repairs), midurethral slings, and perineorrhaphy procedures were all permitted according to attending choice.

The first post-operative trial of void was performed by perioperative nurses with retrograde installation of $300 \mathrm{cc}$ of normal saline after confirmation of anesthesia recovery and resolution of post-operative nausea. Patients were given up to 20 min to void. Voiding trial success was defined as: 1) a postvoid residual less than $150 \mathrm{cc}$ or 2 ) an indication in the chart that the patient had passed the voiding trial in instances when the voiding trial specifics were not recorded but the chart stated that the patient passed her voiding trial and was discharged without need for catheterization.

Study data were collected and managed using a secure Excel file. Abstracted data included demographic characteristics, pertinent comorbidities, smoking status, preoperative Pelvic Organ Prolapse Quantification examination, preoperative residual volume measurement, preoperative presence of stress or urge incontinence, and date of surgery. The operative report, pathology reports, hospital progress notes, and available outside records in our electronic medical system were reviewed to confirm the apical procedures and concomitant surgeries, estimated blood loss, intraoperative complications, postoperative complications, number of days in the hospital, emergency department visits, and unscheduled clinic visits. The outcome of the first voiding trial and any subsequent trials were recorded. If needed, the second voiding trial was typically performed in the outpatient setting after the patient confirmed that they had passed a bowel movement. 
Patients were instructed to remove their catheter at home and if they were unable to void within $4 \mathrm{~h}$, they were instructed to come into the clinic for self-catheterization teaching. Outcome data from postoperative clinic notes and laboratory reports within the study period were also abstracted, including culture-positive urinary infections and the development of long-term urinary disorders including stress incontinence, urge incontinence, mixed incontinence, bladder pain, and urinary retention. An internal random audit of $25 \%$ of charts was performed to confirm accuracy of data abstraction.

Sealedenvelope.com was used to determine the necessary sample size to demonstrate a potential difference in rates of POUR for this study. Using internal institution metrics of approximately $50 \%$ failure rate of first voiding trial for vaginal repair surgery and a $25 \%$ failure rate for SCP, with a $p$ value of 0.05 and $90 \%$ power, the required sample size was 46 patients in each arm. The collected data were exported to $\mathrm{R}$ (RStudio, PBC, Boston, MA) for analysis. A separate study team member from those responsible for data abstraction performed the data analysis. Categorical variables were analyzed using $\chi^{2}$ tests and Fisher exact tests. Continuous variables were analyzed using $t$ test. Multivariate analysis was also completed to control for potential confounders which included variables from the univariate analysis that showed a significant difference, including apical repair technique, anterior repair, posterior repair, anti-incontinence procedure, age, hysterectomy, and age-adjusted Charlson comorbidity index (ACCI) score. It also included variables that could possibly act as confounding variables, including ethnicity and body mass index (BMI). A $P$ value of $\leq 0.05$ was considered statistically significant.

\section{Results}

There were 193 women identified using the specified Current Procedural Terminology codes for the study period. Of these, 44 were excluded because of undergoing a postoperative voiding trial on day greater than 1 , insufficient documentation regarding the results of their first voiding trial, intraoperative complication necessitating prolonged catheterization or concomitant colorectal procedure performed. An additional 11 subjects were excluded because patients were discharged home without specific documentation that a voiding trial was performed. The remaining 134 women were included in the final analysis: 71 in the native tissue repair group (40 sacrospinous fixation and 31 uterosacral suspension) versus 63 in the SCP group (17 laparoscopic and 46 robotic).

Demographic and surgical data is presented in Table 1. The groups were overall similar in characteristics, except that the vaginal repair group was slightly older $(65.45 \pm 12.19$ vs. $61.46 \pm 8.50 p=0.029)$ and had a higher Charlson comorbidity index $(3.00(1.50-3.00)$ vs. $2.00(1.00-2.50) p=$
0.006) (Table 1). The rate of concomitant hysterectomy was $42.3 \%$ in the native tissue and $34.9 \%$ in the SCP groups ( $p=$ -0.385 ). All patients in the SCP group received general anesthesia and in the vaginal group, two patients received spinal anesthesia while the rest received general anesthesia. Twentythree $(32.3 \%)$ patients in the vaginal group had their first postoperative voiding trial on postoperative day 0 while 29 (46.0\%) patients in the SCP group had their first postoperative voiding trial on postoperative day $0(p=0.106)$.

The failure rate of the first postoperative voiding trial was significantly higher in the vaginal repair group (34 vs. $11.1 \%$, $p<0.01$; odds ratio 4.91 ; 95\% CI 1.96-12.3). Both groups had a similar success rate of a second voiding trial $(100 \%$ in SCP group vs. $95.7 \%$ in the vaginal repair group, $p=1$ ). Univariate analysis demonstrated a significantly lower rate of failing the first voiding trial in patients that had a concomitant hysterectomy (odds ratio 0.268 ; $95 \%$ CI $0.088-0.818$ ). Multivariate analysis was performed to control for potential confounding factors associated with postoperative urinary retention as well as factors that were statistically different between groups. These factors included age, ethnicity, BMI, Charlson comorbidity index, anterior repair, posterior repair, sling, high perineorrhaphy, hysterectomy, and length of surgery. In this analysis, there were no variables associated with a higher risk of failing the first postoperative voiding trial aside from the type of procedure and hysterectomy (Fig. 1). The adjusted odds ratio after multivariate analysis for vaginal repair vs. SCP on failing the first postoperative voiding trial was 4.90 (95\% CI 1.91-12.6, $p<0.01$ ).

The total number of days to return of bladder function and postoperative urinary tract infections were significantly higher in the vaginal repair group (3.108 vs. 1.603 days, $p<0.01$ and 23.9 vs. $6.35 \%, p<0.01$ respectively.) Emergency department visits within the first 30 postoperative days were also higher in the vaginal repair group $(15.5$ vs. $1.59 \%, p<0.01)$ and were more likely related to urinary tract concerns $(7 / 11(64 \%)$ vs. $0 / 1(0 \%))$. Five emergency visits in the vaginal group were due to urinary retention and two were due to UTI. Two visits in that group were due to chest pain; one was due to incisional concerns with no sequelae; and one was for dizziness. In the SCP group, the only visit back to the ER was due to chest pain.

\section{Discussion}

In this retrospective cohort study, we demonstrate a significantly greater chance of passing the first postoperative voiding trial in women undergoing a minimally invasive meshaugmented repair compared to native tissue transvaginal repair: These women were almost five times more likely to be discharged postoperatively without the need for ongoing bladder drainage which translated into fewer urinary tract 
Table 1 Demographic and surgical characteristics

\begin{tabular}{|c|c|c|c|}
\hline & $\begin{array}{l}\text { Sacrocolpopexy } \\
(n=63)\end{array}$ & $\begin{array}{l}\text { Native tissue repair, vaginal } \\
(n=71)\end{array}$ & $P$ value \\
\hline \multicolumn{4}{|l|}{ Patient characteristics } \\
\hline Age* & $61.46 \pm 8.50$ & $65.45 \pm 12.19$ & 0.029 \\
\hline Number of delivered children** & $2.00(1.00-3.00)$ & $2.00(1.00-3.00)$ & 0.3736 \\
\hline BMI $\left(\mathrm{kg} / \mathrm{m}^{2}\right)^{*}$ & $27.18 \pm 4.60$ & $28.00 \pm 5.52$ & 0.3545 \\
\hline Tobacco use, current & $8(12.7 \%)$ & $5(7.0 \%)$ & 0.270 \\
\hline Ethnicity & & & 0.628 \\
\hline Caucasian & 53 & 59 & \\
\hline African-American & 5 & 11 & \\
\hline Hispanic & 0 & 0 & \\
\hline Other & 5 & 1 & \\
\hline Diabetes mellitus & $12(19.0 \%)$ & $15(21.1 \%)$ & 0.765 \\
\hline Charlson comorbidity index $* *$ & $2.00(1.00-2.50)$ & $3.00(1.50-3.00)$ & 0.006 \\
\hline Preoperative recurrent UTIs & $9(14.3 \%)$ & $7(9.86 \%)$ & 0.430 \\
\hline \multicolumn{4}{|l|}{ Prior surgical history } \\
\hline Any prior abdominal or pelvic surgery & $55(87.3 \%)$ & $56(78.9 \%)$ & 0.197 \\
\hline Stress incontinence procedure & $8(12.7 \%)$ & $8(11.3 \%)$ & 0.799 \\
\hline \multicolumn{4}{|l|}{ Preoperative exam } \\
\hline Preoperative prolapse stage $(\mathrm{I}-\mathrm{IV})^{* *}$ & $3.00(2.00-3.00)$ & $3.00(2.00-3.00)$ & 0.652 \\
\hline Preoperative stress urinary incontinence & $31(49.2 \%)$ & $32(45.1 \%)$ & 0.409 \\
\hline Preoperative PVR* & $67.03 \pm 84.33$ & $65.98 \pm 77.33$ & 0.941 \\
\hline Preoperative urge urinary incontinence & $27(42.9 \%)$ & $24(33.8 \%)$ & 0.281 \\
\hline \multicolumn{4}{|l|}{ Surgical characteristics } \\
\hline Operative duration (min)* & $217.32 \pm 67.44$ & $122.35 \pm 48.89$ & $<0.01$ \\
\hline Blood loss $(\mathrm{ml})^{*}$ & 92.2561 .27 & 92.5865 .97 & 0.951 \\
\hline Anterior repair & $0(0 \%)$ & $41(57.7 \%)$ & $<0.01$ \\
\hline Posterior repair & $43(68.3 \%)$ & $50(70.4 \%)$ & 0.786 \\
\hline Uterosacral ligament suspension & $0(0 \%)$ & $32(45.1 \%)$ & $<0.01$ \\
\hline Sacrospinous ligament suspension & $0(0 \%)$ & $39(54.9 \%)$ & $<0.01$ \\
\hline Hysterectomy performed & $22(34.9 \%)$ & $30(42.3 \%)$ & 0.385 \\
\hline Stress incontinence procedure performed & $29(46.0 \%)$ & $29(40.8 \%)$ & 0.545 \\
\hline Length of stay (days)** & $1.00(1.00-2.00)$ & $1.00(1.00-1.00)$ & $<0.01$ \\
\hline Persistent PVR $>150 \mathrm{cc}$ greater than 4 weeks & $1(1.59 \%)$ & $2(2.82 \%)$ & 0.631 \\
\hline
\end{tabular}

*Mean \pm standard deviation

**Median (range)

infections and emergency department visits. The pathologic mechanism for this observed difference is not entirely clear, but may be related to higher rates of pelvic floor tension secondary to pain in the native tissue repair group or greater disruption of local autonomic nerves with split-thickness vaginal wall dissections [14]. Interestingly, we did not observe any association between mid-vaginal repairs (anterior or posterior repairs) or mid-urethral slings and postoperative acute urinary retention.

Our results corroborate those of Yune et al., who also demonstrated a significantly higher rate of POUR in women undergoing native tissue apical repair compared to minimally invasive sacrocolpopexy. Their study was limited to comparison between uterosacral suspension and sacrocolpopexy and did not include any sacrospinous fixation, a common method of apical repair. In addition, the authors did not report any follow-up data to determine if there were differences in delayed cases of urinary retention or catheterrelated complications like urinary tract infection. Unlike our cohort, they reported that older age was a significant risk factor for POUR [4]. Our cohort of women undergoing native tissue repair was a mean of 4 years older than the minimally invasive group and this could have further compounded the association. However, when we controlled for age in the multivariable logistic regression analysis, the association of the surgical approach was even stronger. 
Fig. 1 Odds ratio of different variables as they relate to POUR

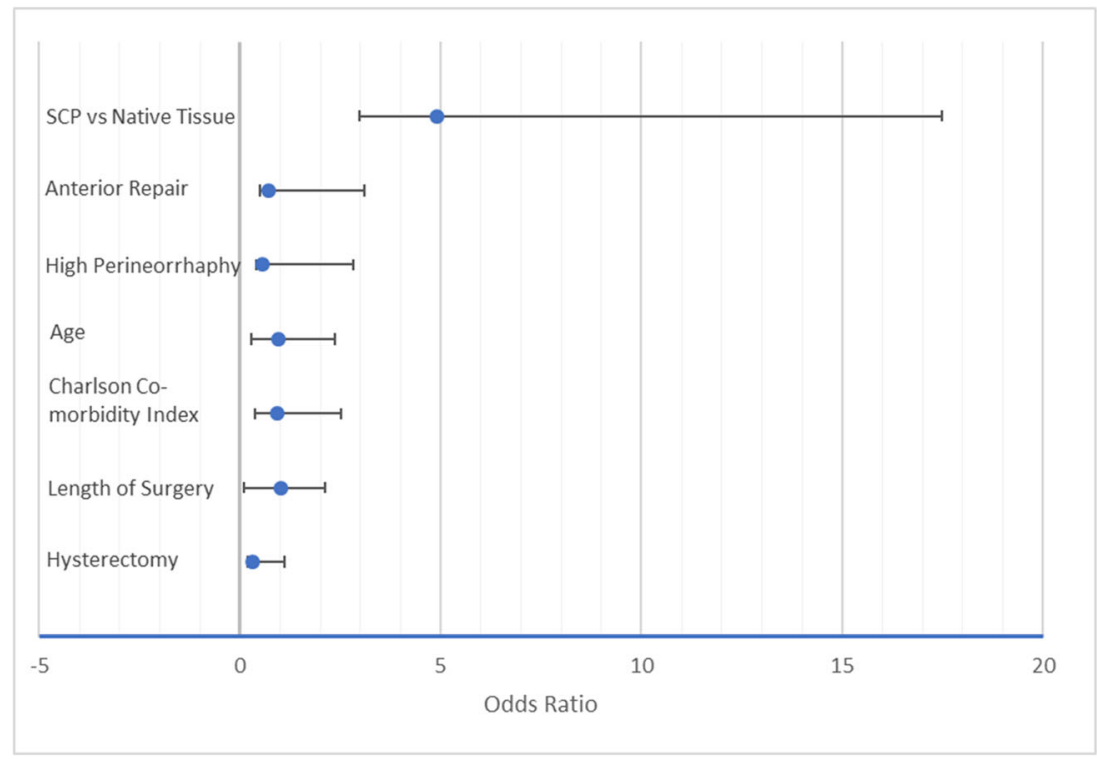

The implementation of an enhanced recovery after surgery (ERAS) protocol may have improved voiding function in both of our study groups. Overall, only $23 \%$ of our cohort failed their first active voiding trial compared to $70 \%$ of women undergoing multi-compartment vaginal native tissue repair at our institution prior to ERAS implementation (3). Similarly, in a retrospective study of 360 patients who underwent apical repair via uterosacral ligament suspension, sacrospinous ligament fixation or robotic sacrocolpopexy, the overall failure rate was $39 \%$ (2). ERAS protocols typically advocate for the incorporation of standing doses of anti-inflammatory medication and acetaminophen without utilization of narcotics, a regimen that improves pain without interfering with return of bladder function.

Delayed return of bladder function in the native tissue group corresponded significantly with urinary tract infection rates and the need for more emergency department visits for bladder-related concerns. Data gleaned from this study can assist with patient-centered prioritization of goals in determining preference for one mode of prolapse repair compared to another. While one patient may be mesh-averse, another may prioritize the opportunity to return home on the day of surgery without the need for bladder drainage with this associated morbidity.

Our study is limited by the retrospective design and potential selection bias for the procedures that could confound the results. The sample of patients chosen was a convenience sample to power the study appropriately and this may decrease external validity of the results. We acknowledge that women in the native tissue apical suspension group were older and had a higher incidence of medical comorbidities compared to the sacrocolpopexy group, variables which can independently affect post-operative bladder function. The first voiding trial was also not performed within an exact window post-surgery (for example within $6 \mathrm{~h}$ ) and therefore, time to first trial could have been a confounding variable. We only included women who had a trial performed within the "23-h" admission window and a total of 65 patients (49\%) were discharged on the same day. There was no statistically significant difference between same-day discharge in the vaginal group and in the SCP group (32 patients $(45 \%)$ vs. 33 patients $(52 \%) p=$ $0.34)$. We were also underpowered to detect differences between patients who underwent uterosacral ligament suspension versus sacrospinous ligament suspension and collectively grouped them into native tissue repair. A larger study is needed to determine if there is indeed a difference between the two types of apical vaginal repairs that were included in this study. Finally, our study is limited to a single institution and therefore may not have external validity. The strengths primarily lie in collection of contemporaneous data following adoption of an enhanced recovery after surgery protocol which has direct applicability for patients planning same day hospital discharge. In addition, evaluation of the association between catheter days, urinary tracts infections, and emergency department visits strengthened the implications of our study findings that acute POUR has more adverse event association than just patient disappointment and discomfort.

In conclusion, we demonstrate that in women participating in an enhanced recovery after surgery protocol, rates of POUR were almost five-fold less in those undergoing sacrocolpopexy as compared to native tissue apical vaginal repair. Early return of bladder function may have also decreased urinary tract-related complications in the first 6 weeks after surgery. Surgeons can use these data to inform preoperative medical decision-making and post-operative patient expectations and goals.

Author contributions AS El Haraki: IRB application, data analysis and manuscript writing. 
J Burns: Data collection.

CL Crafton: Data collection.

C Parker-Autry: Project development.

CA Matthews: Principal investigator, project development, manuscript editing.

\section{Declarations}

Financial conflict of interest/disclosure Catherine Matthews has grant support to institution from Boston Scientific Corporation and Neomedic; Catherine Matthews is a consultant to Boston Scientific Corporation and has received honoraria from Neomedic. All other authors have no conflicts to disclose.

\section{References}

1. Song XC, Zhu L, Liang S, et al. Changes in voiding function after laparoscopic sacrocolpopexy for advanced pelvic organ prolapse: a cohort study of 76 cases. Int Urogynecol J. 2018;29(4):505-12.

2. Eto C, Ford AT, Smith M, Advolodkina P, Northington GM. Retrospective cohort study on the perioperative risk factors for transient voiding dysfunction after apical prolapse repair. Female Pelvic Med Reconstr Surg. 2019;25(2):167-71. https://doi.org/10. 1097/spv.0000000000000675.

3. Schachar JS, Ossin D, Plair AR, Hurtado EA, Parker-Autry C, Badlani G, Davila GW, Matthews CA. Optimal timing of a second postoperative voiding trial in women with incomplete bladder emptying after vaginal reconstructive surgery: a randomized trial. Retrieved November 12, 2020, from https://pubmed.ncbi.nlm.nih. gov/32502559/.

4. Yune J, Cheng JW, Wagner H, Kim J, Hardesty JS, Siddighi S. Postoperative urinary retention after pelvic organ prolapse repair: vaginal versus robotic transabdominal approach. Neurourol Urodyn. 2018;37(5):1794-800. https://doi.org/10.1002/nau.23526.

5. Hakvoort RA, Dijkgraaf MG, Burger MP, Emanuel MH, Roovers JP. Predicting short-term urinary retention after vaginal prolapse surgery. Neurourol Urod. 2009;28:225-8.
6. Carter-Brooks CM, Du AL, Ruppert KM, Romanova AL, Zyczynski HM. Implementation of a urogynecology-specific enhanced recovery after surgery (ERAS) pathway. Am J Obstet Gynecol. 2018;219(5): 495.e491-495.e410. https://doi.org/10. 1016/j.ajog.2018.06.009.

7. Schiavone MB, Herzog TJ, Ananth CV, Wilde ET, Lewin SN, Burke WM, . . . Wright JD. Feasibility and economic impact of same-day discharge for women who undergo laparoscopic hysterectomy. Am J Obstet Gynecol. 2012;207(5):382.e381-389. https:// doi.org/10.1016/j.ajog.2012.09.014

8. Jennings AJ, Spencer RJ, Medlin E, Rice LW, Uppal S. Predictors of 30-day readmission and impact of same-day discharge in laparoscopic hysterectomy. Am J Obstet Gynecol. 2015;213(3): 344.e341-347. https://doi.org/10.1016/j.ajog.2015.05.014.

9. Kenton K, Pham T, Mueller E, Brubaker L. Patient preparedness: an important predictor of surgical outcome. Am J Obstet Gynecol. 2007;197(6):654.e651-656. https://doi.org/10.1016/j.ajog.2007. 08.059 .

10. TM. Hooton, S. B., RW. Haley, T. H., RA. Garibaldi, J. B., JW. Weinstein, D. M., KR. Daniels, G. L., TD. Averch, J. S.JQ. Pulvino, E. D. (1970) Effect of active versus passive void trials on time to patient discharge, urinary tract infection, and urinary retention: A randomized clinical trial. Retrieved from https://link. springer.com/article/10.1007/s00345-019-03005-0.

11. Wein A, Dmochowski R. Neuromuscular dysfunction of the lower urinary tract. In: Wein A, Kavoussi L, Novick A, et al., editors. Campbell-Walsh urology. tenth ed. Philadelphia: Saunders; 2012.

12. Lingaraj K, Ruben M, Chan YH, Das SD. Identification of risk factors for urinary retention following total knee arthroplasty: a Singapore hospital experience. Singap Med J. 2007;48:213-6.

13. Turner LC, Kantartzis K, Shepherd JP. Predictors of postoperative acute urinary retention in women undergoing minimally invasive sacral colpopexy. Female Pelvic Med Reconstr Surg. 2015;21:3942.

14. Book NM, Novi B, Novi JM, Pulvino JQ. Postoperative voiding dysfunction following posterior colporrhaphy. Female Pelvic Med Reconstr Surg. 2012;18:32.

Publisher's note Springer Nature remains neutral with regard to jurisdictional claims in published maps and institutional affiliations. 